\title{
Static and Dynamic Balance Comparison Between the Involved and Uninvolved Sides in Patients Who had Anterior Cruciate Ligament Reconstruction: One- year Follow-up Study
}

\author{
Jin-seong Kim¹, PT, Moon-young Choi ${ }^{1}$, AT, MSc, Doo-hwan Kong ${ }^{1}$, AT, MSc, Kyu-sung Chung ${ }^{2}$, MD, PhD, \\ Ui-jae Hwang ${ }^{3}$, PT, PhD, Oh-yun Kwon ${ }^{3}$, PT, PhD
}

${ }^{1}$ Sports Medical Center, Seoul Paik Hospital, Inje University College of Medicine, ${ }^{2}$ Department of Orthopedic Surgery, Seoul Paik Hospital, Inje University College of Medicine, Seoul, ${ }^{3}$ Department of Physical Therapy, College of Health Science, Yonsei University, Wonju, Korea

\author{
Article Info \\ Received September 18, 2020 \\ Revised October 22, 2020 \\ Accepted October 22, 2020 \\ Corresponding Author \\ Oh-yun Kwon \\ E-mail:kwonoy@yonsei.ac.kr \\ https://orcid.org/0000-0002-9699-768X
}

\section{Key Words}

Anterior cruciate ligament reconstruction Lower extremity

Postural balance
Background: Anterior cruciate ligament reconstruction (ACLR) causes a reduction in the balance of the lower extremities. Static and dynamic balance were evaluated separately to confirm the decrease in balance in patients underwent ACLR. The commonly used methods include the Biodex Balance System (BBS) for static balance and the $Y$ balance test (YBT) for dynamic balance. No study has evaluated whether the static and dynamic balance of the involved side recovers as much as the uninvolved side one year after ACLR.

Objects: The purpose of this study was to investigate the recovery of static and dynamic balance between the involved and the uninvolved sides.

Methods: The BBS (overall, anteroposterior index, and mediolateral index) and YBT (anterior, posterolateral, and posteromedial) of 58 patients underwent ACLR were measured one year postoperation. Both sides of the BBS and the YBT were compared using the paired t-test.

Results: All the index of the BBS showed no difference between the involved and the uninvolved sides, while all the scores of the YBT showed a significant difference in both sides. The YBT anterior result was $54.64 \pm 5.62 \mathrm{~cm}$ in the involved side and $56.90 \pm 5.41 \mathrm{~cm}$ in the uninvolved side $(p=0.001)$. The YBT posterolateral results were $90.12 \pm 10.51 \mathrm{~cm}$ and $92.34 \pm$ $9.85 \mathrm{~cm}(p=0.013)$. The YBT posteromedial results were $93.72 \pm 8.84 \mathrm{~cm}$ and $96.14 \pm 9.37$ $\mathrm{cm}(\mathrm{p}=0.002)$.

Conclusion: A year after ACLR, the static balance showed no difference, while the dynamic balance showed a significant difference in the involved and the uninvolved sides. The static balance of the involved side recovered as much as the uninvolved side, but the dynamic balance did not. Therefore, dynamic balance training should be considered in the rehabilitation program for patients underwent ACLR.

\section{INTRODUCTION}

After anterior cruciate ligament (ACL) injury, reconstruction is usually performed to regain the preinjury activity level [1]. However, strength, functional outcome, and postural stability are reduced despite ACL reconstruction (ACLR) [1,2]. To recover the function of the lower extremities, clinical score, balance test, and functional performance test have been evaluated after ACLR in a clinical setting [3]. Due to a lack of proprioceptive information resulting from the substitution of ACL by the graft, sensory and motor behavior changes become visible [4]. The change reduces the balance of the patients and results in a decreased functional performance [5]. Therefore, it is important to measure the balance accurately and organize an appropriate neuromuscular training program following ACLR [6].

There are several assessment tools and methods to evaluate body balance, which include the Biodex Balance System (BBS) and Y balance test (YBT) [7]. The BBS is relatively easy to use and it is good for targeting large samples [8]. Previous studies related to ACL injury have used the BBS to evaluate body balance [9-12]. The YBT is also relatively inexpensive, portable, and easy to administer, and it has shown good reliability [7,13]. 
The YBT is used to measure dynamic balance associated with lower extremity injuries such as ACL, patellofemoral pain syndrome, and ankle instability [7,14,15].

Although BBS and YBT are classified as balance tests, the purpose of their evaluation is different. Because the BBS is used to measure static balance and the YBT is used to measure dynamic balance [7], it is necessary to apply both BBS and YBT separately. However, previous studies have reported each of BBS and YBT separately for comparison and correlation [12,15-17]. To the best of our knowledge, no study has confirmed simultaneously whether the static and dynamic balance of the involved side recovers as much as the uninvolved side at one year postoperatively.

The purpose of this study was to investigate the recovery of static and dynamic balance between the involved and the uninvolved sides at one year after ACLR. We hypothesized that static and dynamic balance have no significant difference between the involved and the uninvolved sides. Clinically, the results of this study suggest that static and dynamic balance can be regained fully one year after ACLR.

\section{MATERIALS AND METHODS}

\section{Patients}

The study protocol was approved by Seoul Paik Institutional Review Board (PAIK 2020-06-002) and all the patients that participated in the study gave their informed consent. We reviewed the patients' medical records retrospectively to confirm the clinical characteristics between June 2016 and January 2019. Fifty-eight patients who had agreed to undergo all the required tests were followed up over one year postoperation. Inclusion criteria included the following: (1) who had single- bundle ACLR, (2) who used semitendinosus tendon autograft with an outside-in technique for the anatomic femoral tunnel (3) who were followed over 1 year. Exclusion criteria included the following: (1) a concomitant multiple ligament injury, (2) fracture, (3) meniscus root repair, (4) cartilage repair procedures, (5) osteotomy to correct mechanical alignment, (6) revision ACL reconstruction, (7) subtotal or total meniscectomy, and (8) history of prior knee surgery. All the patients underwent an equal follow-up protocol. BBS and YBT were evaluated J.S.K. who was blind to the procedures following ACL reconstruction after one year.

\section{Surgical Techniques}

All surgical procedures were performed via the outside-in technique using a quadrupled semitendinosus tendon autografts with remnant preservation. The reference points of the femoral tunnel and tibial tunnel were based on anatomic center. The femoral tunnel was made by drilling with a flip cutter. After graft passage, the femoral fixation was achieved using tightrope RT (Arthrex, Naples, FL, USA). After 15 times of cyclic loading of graft, a hydroxyapatite interference screw (S\&N Corp., Andover, MA, USA) was used for tibial fixation. In all the cases, post-tie fixation was performed using a cortical screw for additional tibial fixation.

\section{Postoperative Rehabilitation}

Range of motion exercises were initiated at 3-5 days after surgery and a full range of motion was achieved within six weeks. Tolerable weight-bearing began at one or two days after the surgery. Full weight-bearing was allowed at three weeks. The functional knee brace was worn full extension for three weeks and worn without a limited brace angle. After
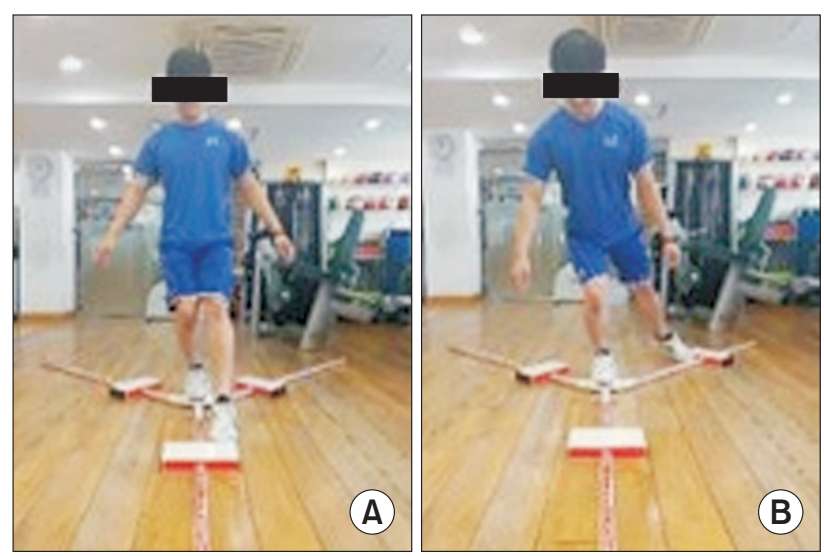

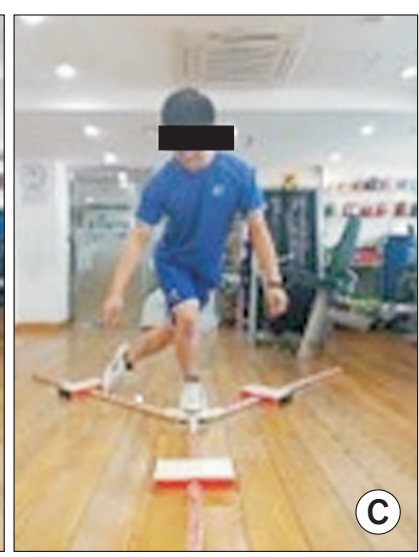

(C)
Figure 1. Y Balance Test directions. (A) Anterior; (B) Posterolateral; (C) Posteromedial. 
then, the brace was taken off 4-6 weeks progressively. The balance training program was initiated progressively from three weeks. At six weeks after surgery, progressively weighted open kinetic chain exercises for quadriceps were allowed within $90^{\circ}-45^{\circ}$ flexion. Three months after the surgery, light running and functional training were initiated progressively. Six months after the surgery, a return to sports with no competition was allowed.

\section{Y Balance Test}

The YBT (Move2Perform, Evansville, IN, USA) was evaluated at one year postoperatively. The score (unit of measurement: $\mathrm{cm}$ ) was measured as the distance of anterior, posterolateral, and posteromedial (Figure 1). The patients stood barefoot on the involved limb and put both hands on their chest with crossing arms. The investigator recorded the third scores after two practices [18] and allowed 10 seconds rest between the trials.

\section{The Biodex Balance System}

The balance was evaluated using the BBS (Biodex Medical System, Shirley, NY, USA) at one year postoperatively (Figure 2). The stability index represents the variance of platform displacement in degrees from level in both the anteroposterior and mediolateral directions. A high number is indicative of poor neuromuscular control, which may increase the potential for orthopedic injury or falling. Overall index, anteroposterior index, and mediolateral index were measured. Level 1 was the

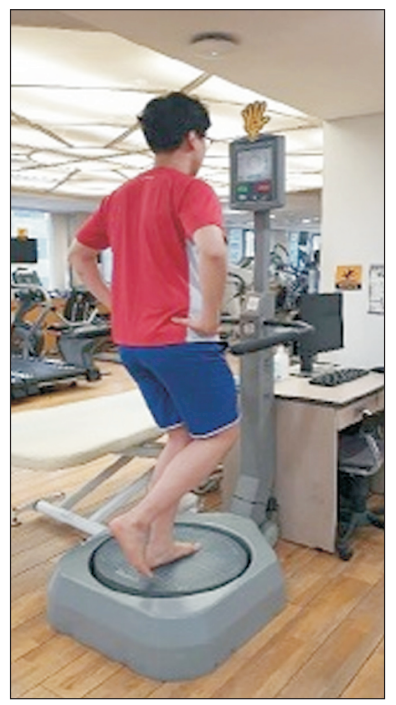

Figure 2. Stability Index of the Biodex Balance System. most unstable and level 12 was the most stable. Patients were instructed to stand barefoot with slight knee bent and their hands placed on their iliac crest and their unsupported limb behind the standing limb. They were then told to maintain their center of pressure on the BBS monitor. Three testing trials were measured for 30 seconds and 10 seconds rest was allowed between trials. The test proceeded at level 8 resistance [19]. During this test, an upright position was demanded on the unstable surface of the BBS.

\section{Statistical Analysis}

All statistical analyses were performed using the SPSS software (ver. 22.0 for Windows; IBM Co., Armonk, NY, USA). The sex, age, height, weight, body mass index, and average final follow-up period were calculated as average and standard deviation to identify the characteristics of the patients. The paired t-test was used to compare the continuous values of the involved and uninvolved sides within the same group. Statistical significance was set at $5 \%(\mathrm{p}<0.05)$.

\section{RESULTS}

The study participants were 45 males and 13 females. Age was $29.6 \pm 8.9$ years. The body mass index was $26.0 \pm 3.6 \mathrm{~kg} / \mathrm{m}^{2}$. The follow-up period was $12.4 \pm 2.0$ months (Table 1).

All index of the BBS showed no significant difference between the involved and uninvolved sides at one year after ACLR. The BBS overall results were $1.54 \pm 0.69$ in the involved side and $1.54 \pm 0.81$ in the uninvolved side $(\mathrm{p}=0.603)$. The BBS anteroposterior index results were $1.06 \pm 0.51$ in the involved side and $1.05 \pm 0.61$ in the uninvolved side $(\mathrm{p}=0.912)$. The BBS-mediolateral index results were $0.81 \pm 0.44$ in involved side and $0.86 \pm 0.52$ in the uninvolved side $(\mathrm{p}=0.350)$ (Table 2).

All the scores of the YBT showed a significant difference

Table 1. General characteristics of included studies ( $N=58$ )

\begin{tabular}{lc}
\hline Patient characteristics & Value \\
\hline Age $(\mathrm{y})$ & $29.6 \pm 8.9$ \\
Sex, male/female & $45 / 13$ \\
Height $(\mathrm{cm})$ & $172.9 \pm 8.5$ \\
Weight $(\mathrm{kg})$ & $78.0 \pm 13.3$ \\
Body mass index $\left(\mathrm{kg} / \mathrm{m}^{2}\right)$ & $26.0 \pm 3.6$ \\
Follow-up period $(\mathrm{mo})$ & $12.4 \pm 2.0$ \\
\hline
\end{tabular}

Values are expressed as mean \pm standard deviation or number only. 
Table 2. Comparison of involved and uninvolved sides ( $N=58)$

\begin{tabular}{lccc}
\hline \multicolumn{1}{c}{ Results } & Involved side & Uninvolved side & p-value \\
\hline $\begin{array}{l}\text { Biodex Balance System } \\
\text { Overall }\end{array}$ & $1.54 \pm 0.69$ & $1.54 \pm 0.81$ & 0.603 \\
$\quad$ Anteroposterior index & $1.06 \pm 0.51$ & $1.05 \pm 0.61$ & 0.912 \\
$\quad$ Mediolateral index & $0.81 \pm 0.44$ & $0.86 \pm 0.52$ & 0.350 \\
Y Balance Test & & & \\
$\quad$ Anterior & $54.64 \pm 5.62$ & $56.90 \pm 5.41$ & $0.001^{*}$ \\
Posterolateral & $90.12 \pm 10.51$ & $92.34 \pm 9.85$ & $0.013^{*}$ \\
Posteromedial & $93.72 \pm 8.84$ & $96.14 \pm 9.37$ & $0.002^{*}$ \\
\hline
\end{tabular}

Values are expressed as mean \pm standard deviation. *Statistically significant at the 0.05 level.

between involved and uninvolved sides at one year following ACLR. The YBT anterior results were $54.64 \pm 5.62 \mathrm{~cm}$ in the involved side and $56.90 \pm 5.41 \mathrm{~cm}$ in the uninvolved side $(\mathrm{p}=$ 0.001). The YBT posterolateral results were $90.12 \pm 10.51 \mathrm{~cm}$ in the involved side and $92.34 \pm 9.85 \mathrm{~cm}$ in the uninvolved side $(\mathrm{p}=0.013)$. The YBT posteromedial results were $93.72 \pm$ $8.84 \mathrm{~cm}$ in the involved side and $96.14 \pm 9.37 \mathrm{~cm}$ in the uninvolved side $(\mathrm{p}=0.002)($ Table 2$)$.

\section{DISCUSSION}

Our study compared the involved and uninvolved sides of YBT and BBS that were measured in patients who had ACLR after a one-year follow-up. We found that there was no difference in the static balance, while the dynamic balance showed a significant difference. The results would mean that the static balance of the involved side at one year following ACLR recovered as much as the uninvolved side, but the dynamic balance did not. Therefore, the dynamic balance could be included in the rehabilitation programs for patients with ACLR at a oneyear follow-up.

The balance provides information about movement, intermediate joint position sense, and control muscle reflex stabilization [10,20]. However, after ACLR, decreased joint position perception, a higher threshold for detection of passive knee motion, longer latency of hamstring muscles, and decreased performance in postural control were shown due to the damage of the ACL mechanoreceptors [4]. Thus, because proprioception of damaged ACL could not recover fully, the training of balance recovery should be performed. Akbari et al. [9] reported that balance training should be part of the rehabilitation program following ACLR. In contrast, Cooper et al. [11] reported that there was no difference between balance training and strength training in the early phase of rehabilitation following ACLR. Although still controversial, the importance of balance recovery has been increasingly emphasized, and it is important to accurately understand the patient's condition. The BBS and YBT are commonly used as tools to evaluate balance. Both of them measure balance, but the purpose of their evaluation is different. Almeida et al. [7] reported that YBT does not correlate with BBS because the YBT is a more dynamic test than the BBS. Therefore, when clinicians evaluate balance, both YBT and BBS need to be used.

The BBS is used to evaluate the balance of several diseases such as ACLR, patellar femoral pain syndrome, chronic lower back pain, and ankle instability [12,14,21,22]. BBS is particularly useful for patients who have undergone ACLR. Mohammadirad et al. [12] reported that BBS has high reliability as a postural stability measurement and is an appropriate balance test for patients who had ACLR. Thus, BBS has been used to confirm the recovery of static balance following ACLR. Mattacola et al. [2] reported that the balance of the involved side in 20 patients who underwent ACLR at average 18 months followup was not significantly different compared to the control group and the uninvolved side, which is consistent with our findings. We found that there was no difference in BBS between the involved and uninvolved sides at one year following ACLR. Therefore, it could be considered that the static balance of the involved side was restored as well as the uninvolved side at one year postoperatively.

Our study also found that YBT was significantly different for the involved and the uninvolved sides in patients who had ACLR after one year. The result could mean that the dynamic balance has not been regained yet. The hamstring muscles are synergistic to ACL and they increase dynamic stability. Thus, the muscles play a very important role in dynamic balance in rehabilitation following ACLR [23]. Recently, several surgeons have chosen hamstring autograft, which is the only semitendinosus tendon or semitendinosus and gracilis tendons, for ACLR [24]. Regeneration of the harvested hamstring is known to be approximately 80 percent [25]. Although the hamstring can be regenerated, knee flexion strength of the involved side using hamstring autograft is reduced compared to the uninvolved side and the involved side using allograft [24]. In our study, dynamic balance did not recover after one year and this was the reason why patients who had ACLR using hamstring autograft had hamstring weakness, which decreases dynamic stability. 
Therefore, strength training and neuromuscular training for hamstring could be included in the rehabilitation program for patients who have undergone ACLR in order to enhance their dynamic balance.

In our study, the YBT was statistically different, but the scores were only 2-3 cm. However, Shaffer et al. [20] reported that the standard of potentially increased risk for injury and asymmetry was more than $4 \mathrm{~cm}$, and the standard used by Gonell et al. [26] for dividing the players into injury group was 4 $\mathrm{cm}$. There was a slight difference from our study and further study will be needed regarding the standard of asymmetry and injury.

A valuable point of our study is that we compared the YBT and BBS between the involved and the uninvolved sides at one year in patients who underwent ACLR. Our study demonstrated that BBS had no significant difference, YBT showed a significant difference at one year postoperatively. The clinical relevance of this study is that patients who undergo ACLR need dynamic balance training until one year postoperation.

There are some limitations to this study. First, a selection bias may have been present because of a retrospective study. Second, the sample size was small. Third, the activity level of the patients who had ACLR was not considered. Thus, the result of the evaluations might be different depending on the ability of patients. Fourth, the follow-up duration was short (one year).

\section{CONCLUSIONS}

After ACLR at one year, the BBS showed no difference, while the YBT showed a significant difference between the involved and the uninvolved sides. The static balance of the involved side following ACLR at one year recovered as much as the uninvolved side, but the dynamic balance did not. Therefore, hamstring muscle training should be included in the rehabilitation program for patients who have undergone ACLR in order to enhance dynamic balance.

\section{ACKNOWLEDGEMENTS}

This study was supported by the "Brain Korea 21 FOUR Project", the Korean Research Foundation for Department of Physical Therapy in the Graduate School of Yonsei University.

\section{CONFLICTS OF INTEREST}

No potential conflict of interest relevant to this article was reported.

\section{AUTHOR CONTRIBUTIONS}

Conceptualization: JK, KC, UH, OK. Data curation: JK. Formal analysis: JK, MC, DK, UH, OK. Investigation: JK, MC, DK. Methodology: JK, MC, DK, UH, OK. Project administration: JK, MC, DK, KC. Supervision: UH, OK. Validation: JK, KC, OK. Visualization: JK, MC, DK, UH. Writing - original draft: JK, OK. Writing - review \& editing: $\mathrm{KC}, \mathrm{UH}, \mathrm{OK}$.

\section{ORCID}

Jin-seong Kim, https://orcid.org/0000-0002-6960-3593

Moon-young Choi, https://orcid.org/0000-0002-1381-1166

Doo-hwan Kong, https://orcid.org/0000-0002-8140-9937

Kyu-sung Chung, https://orcid.org/0000-0002-2378-0359

Ui-jae Hwang, https://orcid.org/0000-0002-2050-5503

\section{REFERENCES}

1. Barber-Westin SD, Noyes FR. Factors used to determine return to unrestricted sports activities after anterior cruciate ligament reconstruction. Arthroscopy 2011;27(12):1697-705.

2. Mattacola CG, Perrin DH, Gansneder BM, Gieck JH, Saliba EN, McCue FC 3rd. Strength, functional outcome, and postural stability after anterior cruciate ligament reconstruction. J Athl Train 2002;37(3):262-68.

3. Chung KS, Ha JK, Ra HJ, Kim SB, Lee GH, Kim JG. Does synovialization after anterior cruciate ligament reconstruction have a positive effect on functional performance, outcomes scores, stability and muscle strength? A 2-year follow-up study after reconstruction. Arch Orthop Trauma Surg 2017;137(12): 1725-33

4. Bonfim TR, Jansen Paccola CA, Barela JA. Proprioceptive and behavior impairments in individuals with anterior cruciate ligament reconstructed knees. Arch Phys Med Rehabil 2003;84(8):1217-23.

5. Fitzgerald GK, Lephart SM, Hwang JH, Wainner RS. Hop tests as predictors of dynamic knee stability. J Orthop Sports Phys Ther 2001;31(10):588-97. 
6. Risberg MA, Holm I, Myklebust G, Engebretsen L. Neuromuscular training versus strength training during first 6 months after anterior cruciate ligament reconstruction: a randomized clinical trial. Phys Ther 2007;87(6):737-50.

7. Almeida GPL, Monteiro IO, Marizeiro DF, Maia LB, de Paula Lima P0. Y balance test has no correlation with the Stability Index of the Biodex Balance System. Musculoskelet Sci Pract 2017;27:1-6.

8. Chen TH, Chou LW, Tsai MW, Lo MJ, Kao MJ. Effectiveness of a heel cup with an arch support insole on the standing balance of the elderly. Clin Interv Aging 2014;9:351-6.

9. Akbari A, Ghiasi F, Mir M, Hosseinifar M. The effects of balance training on static and dynamic postural stability indices after acute ACL reconstruction. Glob J Health Sci 2015;8(4): 68-81.

10. Alonso AC, Greve JM, Camanho GL. Evaluating the center of gravity of dislocations in soccer players with and without reconstruction of the anterior cruciate ligament using a balance platform. Clinics (Sao Paulo) 2009;64(3):163-70.

11. Cooper RL, Taylor NF, Feller JA. A randomised controlled trial of proprioceptive and balance training after surgical reconstruction of the anterior cruciate ligament. Res Sports Med 2005;13(3):217-30.

12. Mohammadirad S, Salavati M, Takamjani IE, Akhbari B, Sherafat S, Mazaheri M, et al. Intra and intersession reliability of a postural control protocol in athletes with and without anterior cruciate ligament reconstruction: a dual-task paradigm. Int J Sports Phys Ther 2012;7(6):627-36

13. Overmoyer GV, Reiser RF 2nd. Relationships between lowerextremity flexibility, asymmetries, and the Y balance test. J Strength Cond Res 2015;29(5):1240-7.

14. Akhbari B, Salavati M, Mohammadi F, Safavi-Farokhi Z. Intraand inter-session reliability of static and dynamic postural control in participants with and without patellofemoral pain syndrome. Physiother Can 2015;67(3):248-53.

15. Myers H, Christopherson Z, Butler RJ. Relationship between the lower quarter Y-balance test scores and isokinetic strength testing in patients status post ACL reconstruction. Int J Sports Phys Ther 2018;13(2):152-9.

16. Yamada RK, Arliani GG, Almeida GP, Venturine AM, Santos CV, Astur DC, et al. The effects of one-half of a soccer match on the postural stability and functional capacity of the lower limbs in young soccer players. Clinics (Sao Paulo) 2012; 67(12):1361-4.

17. Benis R, Bonato M, La Torre A. Elite female basketball players' body-weight neuromuscular training and performance on the Y-balance test. J Athl Train 2016;51(9):688-95.

18. Linek P, Sikora D, Wolny T, Saulicz E. Reliability and number of trials of Y balance test in adolescent athletes. Musculoskelet Sci Pract 2017;31:72-5.

19. Arifin N, Abu Osman NA, Wan Abas WA. Intrarater test-retest reliability of static and dynamic stability indexes measurement using the Biodex Stability System during unilateral stance. J Appl Biomech 2014;30(2):300-4.

20. Shaffer SW, Teyhen DS, Lorenson CL, Warren RL, Koreerat CM, Straseske CA, et al. Y-balance test: a reliability study involving multiple raters. Mil Med 2013;178(11):1264-70.

21. Testerman C, Vander Griend R. Evaluation of ankle instability using the Biodex Stability System. Foot Ankle Int 1999;20(5): 317-21.

22. Sherafat S, Salavati M, Ebrahimi Takamjani I, Akhbari B, Mohammadirad S, Mazaheri M, et al. Intrasession and intersession reliability of postural control in participants with and without nonspecific low back pain using the Biodex Balance System. J Manipulative Physiol Ther 2013;36(2):111-8.

23. Henriksson M, Ledin T, Good L. Postural control after anterior cruciate ligament reconstruction and functional rehabilitation. Am J Sports Med 2001;29(3):359-66.

24. Kim JG, Yang SJ, Lee YS, Shim JC, Ra HJ, Choi JY. The effects of hamstring harvesting on outcomes in anterior cruciate ligament-reconstructed patients: a comparative study between hamstring-harvested and -unharvested patients. Arthroscopy 2011;27(9):1226-34.

25. Choi JY, Ha JK, Kim YW, Shim JC, Yang SJ, Kim JG. Relationships among tendon regeneration on MRI, flexor strength, and functional performance after anterior cruciate ligament reconstruction with hamstring autograft. Am J Sports Med 2012;40(1):152-62.

26. Gonell AC, Romero JA, Soler LM. Relationship between the Y balance test scores and soft tissue injury incidence in a soccer team. Int J Sports Phys Ther 2015;10(7):955-66. 